\title{
Narrativa
}

\section{L'estinzione della specie maschile: Caro Michele di Natalia Ginzburg}

\section{Marco Antonio Bazzocchi}

\section{(2) OpenEdition}

\section{Journals}

\section{Edizione digitale}

URL: https://journals.openedition.org/narrativa/542

DOI: $10.4000 /$ narrativa. 542

ISSN: 2804-1224

\section{Editore}

Presses universitaires de Paris Nanterre

\section{Edizione cartacea}

Data di pubblicazione: 1 décembre 2018

Paginazione: 105-116

ISBN: 978-2-84016-325-1

ISSN: $1166-3243$

\section{Notizia bibliografica digitale}

Marco Antonio Bazzocchi, «L'estinzione della specie maschile: Caro Michele di Natalia Ginzburg», Narrativa [Online], 40 | 2018, online dal 01 novembre 2021, consultato il 08 décembre 2021. URL: http://journals.openedition.org/narrativa/542 ; DOl: https://doi.org/10.4000/narrativa.542

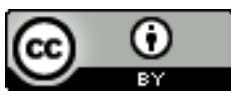

Narrativa est mise à disposition selon les termes de la Licence Creative Commons Attribution 4.0 International. 


\title{
L'estinzione della specie maschile: Caro Michele di Natalia Ginz̧urg
}

\begin{abstract}
RIASSUNTO
Concentrandosi su Caro Michele di Natalia Ginzburg, il saggio di Bazzocchi mette in evidenza la frammentazione di una famiglia borghese e il progressivo indebolimento del ruolo maschile tradizionale. Michele, protagonista in absentia, mostra un'identità sessuale indefinita (passando, nel corso del romanzo da eterosessuale ad omosessuale a bisessuale) e un rifiuto di assumere il ruolo di pater familias. La tradizionale dicotomia maschile/femminile viene inoltre scompaginata nei rapporti che si creano tra gli altri personaggi del romanzo.
\end{abstract}

\section{RÉSUMÉ}

En ce concentrant sur Caro Michele de Natalia Ginzburg, cette étude souligne la fragmentation d'une famille bourgeoise et l'affaiblissement progressif du rôle masculin traditionnel. Michele, protagoniste in absentia, présente une identité sexuelle indéfinie (passant, tout au long du roman, d'hétérosexuel à homosexuel à bisexuel) et il refuse d'assumer le rôle de pater familias. La dichotomie traditionnelle entre masculin et féminin est par ailleurs brouillée dans les rapports qui s'établissent entre les autres personnages du roman.

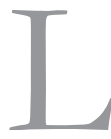

'inizio degli anni settanta coincide con una svolta nella rappresentazione della sessualità nel romanzo italiano. Pensare questa svolta nei termini di maschile o femminile renderebbe molto semplice l'indagine, mentre invece sembra più sensato considerare la tensione (o lo scarto) che si crea tra le due polarità. L'esempio che sarà qui analizzato potrebbe costituire un punto di partenza per ridisegnare una mappa letteraria secondo principi non solo formali. Natalia Ginzburg consegna alle stampe nel 1973 Caro Michele quando ormai il romanzo tradizionale degli anni sessanta ha esaurito quasi tutte le sue potenzialità e si sono affacciate altre sperimentazioni letterarie. Elsa Morante, modello 
inarrivabile di scrittrice cui Ginzburg guarda con ammirazione, non ha ancora pubblicato Aracoeli, che uscirà come un meteorite anomalo solo all'inizio degli anni ottanta, e contiene una specie di blasfemia progressiva nei confronti della maternità, con un improvviso capovolgimento finale che esalta invece l'amore per il padre da parte di un figlio omosessuale. In Ginzburg i ruoli maschili e femminili sembrano ancora abbastanza stabili, e i rapporti interni alla famiglia analizzati con la prospettiva di chi è già passato attraverso il primo grande sconvolgimento del 1968. Il romanzo è, infatti, il frutto della fine di quel mondo, oramai proiettato verso una nuova realtà. Vi si analizza il disfacimento di una famiglia, dalla quale sono stati generati i grappoli di altri nuclei famigliari. Ma in tutti i casi sembra che una incrinatura all'interno della famiglia di origine si sia prolungata nelle famiglie satellite, con un procedimento inarrestabile. Se c'è un elemento che Ginzburg sottopone a un'analisi attenta e a volte spietata è proprio il mondo maschile, dove sembra regnare l'assoluta incapacità di tenere insieme $i$ frammenti che invece le donne cercano di salvare da una specie di day-after, come notarono Fruttero e Lucentini nella recensione al romanzo, Natalia e le cose.

Se nella Morante l'affettività si gioca sulla linea figlio/madre, e la posizione del padre è sempre collaterale, anche perché sul padre cade a volte un sospetto di omosessualità (come avviene per Wilhelm Gerace nell'Isola di Arturo), Ginzburg utilizza l'omosessualità come elemento non esplicito, allusivo, che però rivela in qualche modo una profonda implicazione con il mondo materno. In Caro Michele assistiamo al percorso con cui una madre acquista consapevolezza del rapporto col proprio figlio anche attraverso il personaggio di un presunto omosessuale maturo (Osvaldo) che potrebbe esser stato l'amante di Michele, e a cui il ragazzo ha dedicato comunque molta attenzione. Lo stesso avviene per Angelica, una delle due sorelle di Michele, che compie un processo di crescita anche attraverso la presenza di Osvaldo. L'omosessualità in Ginzburg è sempre mantenuta sottotraccia, non è mai una rivelazione drammatica come nella Morante, e soprattutto l'omosessualità non diventa una reazione violenta a traumi subiti nell'orbita della madre. In un certo senso possiamo pensare l'omosessualità maschile come una presenza intima di supporto al mondo femminile, una presenza di supporto che nel romanzo inizia sottotono e trova invece piena realizzazione nel finale.

Cesare Garboli si era domandato quale sia la "specie femminile"1 cui appartiene l'autrice. La terminologia usata suggerisce innanzitutto che il mondo

1. Garboli Cesare, Prefazione a Ginzburg Natalia, Caro Michele, Torino, Einaudi 2001, p. V. 
femminile, osservato con singolare attenzione da Garboli, è composto da tante realtà differenti, tra le quali non è facile distinguere quella di appartenenza da Ginzburg:

Inutile cercare vicino a noi. Bisogna risalire indietro nel tempo, lavorare di fantasia, immaginarsi un remoto va e vieni di gente nomade nel deserto, quando non erano rari gli esemplari di donne dai nomi antichi come Sara, esotici come Abigail: donne tutte di un pezzo, il passo abituato alle lunghe marce, il busto eretto sotto il carico di masserizie e di figli. Guerre, calamità, carestie si avvicendavano senza scuotere il ritmo quotidiano della loro esistenza; senza alternare le abitudini elementari: attendarsi, accendere il fuoco, cucinare, mangiare, partorire, prendere sonno ${ }^{2}$.

Garboli rimanda a un'altra cultura e a un'altra epoca, in cui gli individui vivevano all'interno di comunità, definite tribù, e le donne (dai nomi esotici) erano creature resistentissime: attraversavano il deserto, le guerre e le carestie. Anche nelle opere precedenti come Lessico famigliare (Einaudi, 1963) o I rapporti umani (contenuto in Le piccole virtù, Einaudi, 1953), Ginzburg rappresenta storie di famiglie, all'apparenza borghesi, ma che in realtà sono vere e proprie tribù, in cui sopravvive qualcosa di arcaico, elementare e lontano. Ma sembra che sull'omosessualità cada una specie di divieto, o di non detto (qualcosa di simile agisce anche in Debenedetti quando parla dell'Isola di Arturo).

Nella prima lettera Adriana (la madre di Michele) confessa al figlio la paura per le malattie e il suo strano rapporto con i rimorsi, in occasione della malattia del marito da cui è ormai separata:

Forse pensi che io dovrei trasferirmi in casa di tuo padre e assisterlo. Anch'io lo penso in qualche momento, ma credo che non lo farò. Ho paura delle malattie. Ho paura delle malattie degli altri, delle mie no, ma io però non ho mai avuto grandi malattie. Quando mio padre aveva la diverticolite, ho fatto un viaggio in Olanda. Ma lo sapevo benissimo che non era diverticolite. Era cancro. Così quando è morto non c'ero. Ne ho rimorso. Ma è vero che a un certo punto della nostra vita i rimorsi li inzuppiamo nel caffè la mattina come biscotti ${ }^{3}$.

In tono antidrammatico, Adriana esprime il fatto di non aver voluto assistere alla morte di suo padre, così come ora specularmente Michele cerca con ogni sforzo di restare lontano da casa quando si presenta la malattia del proprio

2. Ibid.

3. Ibid., pp. 4-5. 
padre. L'immagine semplice e cinica dei biscotti a colazione, che richiama la quotidianità del rimorso con cui si deve convivere, esibisce anche con tagliente elementarità alcune caratteristiche della donna: è dura (almeno all'apparenza) e non ha timore nell'esibire i propri affetti elementari, depurati da qualsiasi forma di sentimentalismo. Adriana ha spezzato un vincolo filiale, sottraendosi alla norma secondo cui era necessario che lei si prendesse cura del padre. Il "prendersi cura di" sembra essere un tabù che circola in tutti i rapporti del romanzo, tanto nelle presenze femminili che in quelle maschili. Michele stesso, il personaggio più muto ed enigmatico, sembra incapace di "prendersi cura" di chiunque. Solo il padre di Michele, nelle parole della madre, è completamente coinvolto dal rapporto con il figlio, tanto da considerarlo l'unica "stella" della sua vita. Nell'Isola di Arturo, Stella è proprio il nome del galeotto di cui è innamorato Wilhelm, padre di Arturo (che a sua volta, oltre a ricordare Rimbaud, ha il nome di una stella, per alludere al legame incestuoso dell'amore paterno).

La situazione narrata nel romanzo rispecchia quella di una famiglia medioborghese che lentamente si sta sfaldando ${ }^{4}$. La stessa topografia, contraddistinta dalla presenza di tre case distinte (quella del padre, della madre e di Michele), attesta come questa sia dispersa e priva di un centro unificatore dove far convergere un comune spazio domestico. Il nomadismo della Prefazione di Garboli deve essere inteso anche secondo quest'ottica: non solo c'è una dispersione del nucleo famigliare ma sussiste anche un movimento continuo tra le diverse abitazioni, che sono nuclei dai quali si diramano i sentimenti residui di tutti i personaggi, incapaci di investire la loro affettività in un unico luogo, in un centro. Ginzburg intende, nello svolgersi delle lettere, sottolineare la gradualità del processo. Al contrario di quanto avviene in Teorema di Pasolini, in cui la famiglia borghese implode al contatto con l'Ospite, in modo improvviso, quella di Ginzburg procede verso il dissolvimento per tappe rallentate, intervallate da ampi momenti di stallo. E se in Teorema tutto il processo di dissolvimento passa attraverso la forza esplosiva dell'eros maschile dell'Ospite, in Ginzburg l'eros è sempre tenuto in sottofondo, o è visto come una realtà pulviscolare che si deposita con molta delicatezza sulla vita degli individui. Non

4. Sulla rappresentazione della famiglia nell'opera della Ginzburg, rimando a quanto dice Domenico Scarpa nella sua postfazione a GINZBURG Natalia, Tutto il teatro, Torino, Einaudi, 2005, in particolare alle pp. 433-434. Per il processo di integrazione dell'omosessuale all'interno del nucleo famigliare si può vedere il percorso abbozzato da ForTney James Michael, "Con quel tipo li: Homosexual Characters in Natalia Ginzburg’s Narrative Families”, Italica, Lxxxvi (2009), 4, pp. 651-673. 
a caso le uniche scene che possono definirsi erotiche sono connesse al personaggio di Mara, che ha caratteristiche completamente diverse da tutti i membri della famiglia.

La fredda lentezza con cui procede il movimento narrativo caratterizza anche i momenti drammatici del testo. Attraverso la particolare tecnica consentita dal genere epistolare, l'autrice, infatti, depotenzia la drammaticità di determinati episodi, inserendoli all'interno delle missive come elementi che i personaggi danno per scontati, ma che in realtà sono determinanti per l'evolversi della vicenda stessa. Il rovesciamento conseguentemente ottenuto relega sullo sfondo gli eventi decisivi, che giungono al lettore come filtrati, e porta in primo piano la dimensione cruda degli affetti e dei sentimenti. Se è vero quanto affermato da Garboli che la Ginzburg appartiene a una specie femminile di antica fattura biblica, anche il suo modo di percepire i fatti è diverso rispetto a quello degli uomini del Novecento. Secondo una vulgata approssimativa i sentimenti fanno parte della sfera emotiva femminile: allora bisogna dire che Ginzburg ha adottato per le sue donne un atteggiamento emotivo in cui il femminile è avvicinato al limite con la maschilità, e questo lo vediamo soprattutto per la figura materna di Adriana.

Adriana viene a conoscenza, per mezzo della mediazione di Osvaldo, che Michele ha lasciato l'Italia ed è partito per Londra, dopo aver brevemente salutato il padre mentre stava dormendo. Da questo momento, Michele non tornerà più in Italia e attraverserà diversi luoghi d'Europa, per trovare la morte durante una manifestazione a Bruges. Michele si colloca all'interno dei movimenti di contestazione giovanile dei tardi anni sessanta, sulla soglia dei cosiddetti "anni di piombo", nonostante non vi siano particolari riferimenti che esplicitino il contesto di riferimento. In particolare, nella lettera la madre lamenta la partenza del figlio e ne domanda le ragioni:

Gli [a Osvaldo] ho chiesto se tu eri partito per via di quella Mara Castorelli che mi ha scritto e che ha un bambino. Osvaldo mi ha detto che quel bambino non è tuo. Secondo lui la ragazza con la tua partenza non c'entra niente. Dice che è solo una povera e balorda ragazza senza soldi, senza una coperta di lana e senza una sedia [...] Ho sempre una paura tremenda che tu possa finire nei tupamaros $^{5}$.

Nonostante usi il termine "balorda" per designare la ragazza, così come prima ha fatto riferendosi a Michele, Adriana non desidera dare un giudizio

5. GinzBurg Natalia, Caro Michele, cit., pp. 24-25. 
morale, ma indica - secondo un'abitudine linguistica che ritorna altre volte tutti quei ragazzi che non sanno cosa fare nella loro vita e non hanno delle idee chiare sul futuro. Quella dei giovani di Caro Michele è una generazione di "balordi" cui si contrappone una certa idea di genitorialità. Tuttavia, dalla lettera si evince come Adriana non sia tanto interessata agli elementi astratti (emotivi) della vicenda quanto alla concretezza, agli aspetti tangibili della quotidianità. La sua attenzione è rivolta agli oggetti: in particolare alla coperta per coprirsi e a una stufa verde che ha regalato al figlio. La distanza tra i due si consuma nel rapporto con gli oggetti: mentre Adriana è affezionata agli oggetti regalati, correlativo oggettivo dell'affetto per il figlio, Michele non se ne preoccupa, anzi non li usa oppure li mantiene sporchi e consunti. "Mi ricordavo del giorno che ero andata a comperartela e perciò mi era cara. Tu certo troverai stupido che uno possa amare una stufa. Osvaldo mi ha detto che tu non la accendevi mai quella stufa perché non ti ricordavi mai di ordinare la legna e ne usavi invece una elettrica".

La figura di Osvaldo ingenera nella donna un particolare fastidio, dovuto principalmente al fatto che non chiarisce i dubbi di Adriana:

Non capisco bene se mi è simpatico. È gentile. È di una tale gentilezza che dà perfino una sensazione di ripienezza, come quando abbiamo mangiato troppa marmellata. Ha quella faccia florida che ride sempre. Ma io non vedo proprio niente da ridere. Guardandolo avevo in qualche momento il dubbio che fosse pederasta. Non ho mai capito bene come mai siete tanto amici, tu un ragazzo, lui un uomo di trentasei o trentotto anni. Ma dirai che il numero delle mie paure per te è illimitato ${ }^{7}$.

Molte volte nel corso del romanzo emerge il sospetto che Osvaldo sia omosessuale ("pederasta" ne è qui un sinonimo, legato all'uso linguistico un po' datato della madre). Adriana, infatti, non solo sospetta della distanza (sociale e di età) tra il figlio e Osvaldo, ma è persuasa del fatto che Michele voglia più bene all'uomo che a lei. Tuttavia nel corso del racconto lo sguardo di Adriana sull'amico adulto del figlio muta, perché Osvaldo inizia a frequentare la casa della donna e diviene il tramite e il mediatore di Michele principale nel rapporto con la famiglia.

La prima missiva di Michele, indirizzata però alla sorella Angelica, si trova nel quarto capitolo. Seppur breve, essa ha lo scopo di ordinare alla ragazza di recarsi

6. Ibid.

7. Ibid. 
nello scantinato (dove viveva Michele) per prelevare dall'interno della stufa a legna un piccolo mitra nascosto e buttarlo nel Tevere: "Questa incombenza non la do a Osvaldo. La do a te. Anzi Osvaldo preferirei non ne sapesse niente. Non voglio che mi giudichi un completo imbecille. Però se invece ti viene voglia di raccontarlo a Osvaldo, raccontaglielo. In fondo, che lui mi trovi un imbecille mi

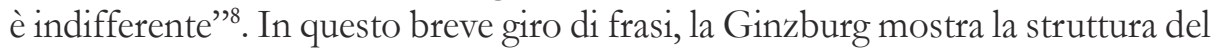
doppio legame del sentimento. A un sentimento di vergogna e di riserbo nei confronti dell'amico si oppone poi una netta indifferenza. In Michele - appare evidente sin da questa prima lettera - non c'è consapevolezza di ciò che fa, sente e pensa. Scappa dall'Italia perché ha paura che la polizia lo colleghi in qualche modo a gruppi sovversivi armati e anche perché è consapevole di essere forse il padre del bambino di Mara. Osvaldo però viene messo sotto una luce particolare: non a caso l'incarico (l'incombenza) passa da lui a Angelica, cioè scivola ma nello stesso tempo lega insieme le due figure.

Da questo momento, Angelica acquista un ruolo centrale. Le tre figure femminili principali (Angelica, Mara e la madre) rappresentano tre diversi aspetti della femminilità che non hanno nulla in comune tra loro. Adriana è problematica, ma è come una quercia dura e solida e si contraddistingue per una cristallina schiettezza attraverso cui si relaziona con i figli. Mara, al contrario, è balorda, giovane e sconsiderata. Angelica, invece, è una figura di intermediaria, costruita sul modello della figura classica di Antigone, che porta su di sé i conflitti e i sentimenti interni alla sua famiglia, elaborandoli sulla base di una propria profonda idea di morale. Antigone è colei che si oppone alla legge pubblica per difendere le ragioni di una legge privata, interna ai legami famigliari. È lei, infatti, che vuole rendere omaggio al cadavere del fratello Polinice, considerato un traditore della città: il legame di sorellanza acquista con lei un valore assoluto, simbolicamente capace di riscattare gli altri legami degradati della famiglia di Edipo (sarà lei ad accompagnare il vecchio Edipo cieco a Colono, il sobborgo di Atene dove Edipo può finalmente trovare una morte giusta). Allo stesso modo, Angelica è l'anello di congiunzione tra la vecchia generazione, incarnata da Adriana, e quella nuova di Michele e Mara. Osvaldo e Angelica costruiscono una rete di relazioni atta a non far disperdere i legami affettivi e a rinsaldare il rapporto tra Michele e la madre. Non a caso la stessa relazione tra Osvaldo e Adriana si consolida al punto da divenire una salda abitudine.

Nel capitolo XVI Angelica incontra la sorella Viola. Le due si soffermano, in particolare, sul fatto che il padre ha lasciato in eredità a Michele la parte più

8. Ibid., p. 29. 
cospicua del patrimonio. Viola desidera per sé e la sua famiglia la torre acquistata dal padre presso l'Isola del Giglio. La torre, al pari di molti altri oggetti, ha un valore simbolico: rappresenta, infatti, per mezzo della sua isolata posizione, le figure del romanzo stesso, separate e divise da una incolmabile distanza. Angelica, al contrario, è fedele alla volontà del padre e non discute la sua scelta testamentaria. È interessante la piega che da questo punto in avanti assume il discorso tra le due sorelle. Viola, infatti, dopo aver descritto come avrebbe trasformato la torre, confida ad Angelica che il fratello non avrebbe mai vissuto in quel luogo e che mai si sarebbe sposato: "È un omosessuale, - disse Viola, non l'avevi capito che lui e Osvaldo erano amanti'. 'Te lo sogni', disse Angelica. Mentre diceva 'te lo sogni' si accorse che invece l'aveva pensato sempre". $\mathrm{La}$ Ginzburg non inserisce il discorso su Michele per un pettegolezzo famigliare, bensì cerca attraverso lo sfogo di Viola di mostrare, al pari di quanto ha fatto la Morante, la crisi di identità in cui si trova il mondo maschile (di "crisi della mascolinità" aveva parlato uno dei primi recensori, Vittorio Spinazzola). L'autrice crea intorno alla figura di Michele l'effetto di un'incertezza sessuale che si riverbera anche sulla sua esistenza e sul suo destino. Nulla vieta di pensare che tra Michele e Osvaldo intercorresse qualche cosa di più di una semplice amicizia, tuttavia avendo avuto entrambi delle donne (e forse anche un figlio) Viola introduce nel discorso il termine "ambidestro", cioè bisessuale. Angelica, con un senso di sfacelo derivato dall'assenza di un nuovo centro maschile nella famiglia, è persuasa del contrario, mentre Viola è sicura che sia stato loro padre a rovinarlo: "Lo adorava e lo viziava. L'ha tolto a noi e alla mamma. Lo trascurava. Lo adorava e lo trascurava. Lo lasciava sempre solo in casa con delle vecchie cuoche. È così che è diventato omosessuale, Michele"10.

Nella terza lettera rivolta a Osvaldo, Michele, dopo aver dichiarato la propria contentezza per l'avvicinamento dell'amico alla madre, fa un riferimento a un oggetto che conferma il rapporto intenso intercorso tra loro:

Nel mio scantinato, credo in fondo a un cassetto del comò, c'è una sciarpa. È una bellissima sciarpa, di vero cachemire, bianca a strisce celesti. Me l'aveva regalata mio padre. Vorrei che tu andassi a cercarla e la portassi. Sarei contento di sapere che hai al collo quella sciarpa, quando cammini per il lungotevere, uscendo dal tuo botteghino. Non ho dimenticato le nostre lunghe passeggiate sul lungotevere, avanti e indietro, col sole che tramontava ${ }^{11}$.

9. Ibid., pp. 83-84.

10. Ibid.

11. Ibid., pp. 90-91. 
L'oggetto, al pari di altri, ha un valore positivo, perché rinsalda l'affettività tra Osvaldo e Michele. L'incomprensione - qui e in pochi altri passi del romanzo - è superata per mezzo di piccoli fili (seppur non risolutivi) capaci di creare rapporti.

La presunta omosessualità di Michele diventa oggetto continuo di interrogazione da parte delle donne di famiglia. Viola rivela alla sorella Angelica che durante l'infanzia nutriva un profondo amore per Michele, tant'è che giocavano persino insieme con le bambole. Viola, presenza incostante del romanzo, ha un'idea precisa del fratello e del suo rapporto con Osvaldo:

"Era un'amicizia di omosessuali. Inutile volerselo nascondere. Si capiva al solo vederli. Me l'ha detto anche Elio, che li ha visti insieme. Io ancora non so darmi pace che Michele fosse diventato un omosessuale. Michele direbbe che sono conformista. Mi dà angoscia di vedere Osvaldo. È gentile, è tutto quello che vuoi, ma a me dà angoscia vederlo. Lo vedrò spesso, perché viene spesso qui. Cosa venga a fare, non si sa. Eccolo. Sta arrivando. Riconosco il rumore della cinquecento. Ma alla mamma fa piacere. $\mathrm{O}$ non ci pensa, oppure ci pensa e si è abituata. Probabilmente ci si abitua a tutto". "Ci si abitua a tutto quando non rimane più niente", disse Angelica ${ }^{12}$.

La battuta chiude qualsiasi considerazione. Ginzburg termina entrambi i capitoli con la parola "niente": tutto ciò che i personaggi si dicono ruota intorno a una mancanza. L'unico personaggio che non manifesta così esplicitamente una mancanza concreta o psicologica è Osvaldo, perché attraversa le vicende sempre come testimone. Ed è proprio la sua discrezione a contenere il residuo di valori che può costituire un poco di salvezza plausibile all'interno della famiglia. Il segreto di Osvaldo, così come il segreto di Michele, perdono però di centralità. Il lettore viene guidato su una prospettiva diversa, che non riguarda più la curiosità sull'effettiva natura delle scelte sessuali dei due personaggi (Chi è realmente Osvaldo? E da cosa sono state motivate le scelte di Michele? Il ragazzo è forse fuggito dalla consapevolezza della propria inclinazione?). Se usassimo la pratica decostruttiva di Eve Kosofsky Sedgwick potremmo ipotizzare che il binarismo sessuale appare nel romanzo per essere poi sorpassato, o reso inefficace, proprio grazie all'ellissi che tocca la sessualità di Osvaldo, e di riflesso quella di Michele ${ }^{13}$. Il nuovo oggetto di attenzione diventa il residuo di

\section{Ibid., p. 153.}

13. Mi riferisco al più volte citato saggio del 1990 che cito dall'edizione francese: Kosofsky SEdgwick Eve, Epistémologie du placard, Éditions Amsterdam, 2008. Per riprendere una recente osservazione di Tommaso Giartosio (Non aver mai finito di dire. 
affettività che Osvaldo e Angelica possono recuperare elaborando insieme il lutto. Ginzburg conduce un'operazione che possiamo leggere con l'aiuto di quelle categorie meno sessualizzate nella genealogia dell'omosessualità di cui appunto parla Kosofky Sedwick e che avvicinerei al discorso di Roland Barthes sul "neutro" come interrogazione su un'etica depotenziata di istanze virili. Al paradigma del "segreto" che accompagna fin dalla fondazione la letteratura omosessuale, Ginzburg sostituisce il tema dell'affetto, che sposta la sessualità su un piano diverso e riesce a legare insieme i personaggi maschili e quelli femminili, anche se è la femminilità a preponderare sulla mascolinità.

Nella lettera conclusiva la Ginzburg dà per l'ultima volta la parola a Osvaldo che scrive ad Angelica. I due mediatori, gli unici due personaggi che si salvano dall'apocalisse inscenata dall'autrice, chiudono l'intera vicenda. Osvaldo si trova a Leeds per compiere un breve pellegrinaggio nell'ultimo luogo dove Michele ha abitato. Nella città incontra Ermanno, l'amico di cui nessuno sa niente e che scrive in Italia per raccontare la fuga del ragazzo verso l'Europa continentale. Dall'incontro Osvaldo scopre che Eileen è tornata insieme con i figli negli Stati Uniti; inoltre che seppur donna di grande intelligenza era un'alcolizzata che Michele si era proposto di salvare. Michele, dunque, si è sposato per un atto di generosità verso una donna che ammirava, ma non amava realmente. All'interno della casa, dove i coniugi hanno vissuto, Osvaldo scopre alcune tracce della loro permanenza: un grembiale da cucina, un impermeabile strappato, delle figure di Biancaneve e i sette nani poste alle pareti e una ciotola di latte abbandonato da un gatto. "Se ti descrivo così accuratamente queste cose, è perché penso che ti è caro saperle"14. In questa frase, la Ginzburg condensa il senso della misericordia, così come viene presentato nel capitolo ventinovesimo. Nel recupero della memoria legato a un atto d'amore per l'altro risiede l'unico modo per potersi salvare in un mondo profondamente mutato:

Di Michele non ho trovato nulla, salvo una camiciola di lana usata come cencio da spolvero e appesa a una scopa, e mi è sembrata una camiciola che lui un tempo si era comperata per l'inverno e infatti ho guardato e c'era la targa di Anticoli, quel negozio in via della Vite. Dopo un attimo d'incertezza,

Classici gay, letture queer, Macerata, Quodlibet 2017) si potrebbe pensare che ci siano testi letterari, come Caro Michele, che non si situano all'interno di grandi mainstream letterari ma che rendono problematiche proprio le categorie identitarie, e lo fanno all'interno di narrazioni dall'apparenza molto convenzionale.

14. Ginzburg Natalia, Caro Michele, cit., pp. 155. 
l'ho lasciata dov'era. Io credo che non serve a niente conservare gli oggetti dei morti, quando sono stati maneggiati da ignoti e si è svaporata la loro identità ${ }^{15}$.

Dopo aver incontrato il ragazzo che ha annunciato alla famiglia la sua partenza, Osvaldo medita sui ragazzi d'oggi, completando il discorso avviato dalla madre intorno alle memorie.

I ragazzi oggi non hanno memoria, e soprattutto non la coltivano, e tu sai che anche Michele non aveva memoria, o meglio non si piegava mai a respirarla e coltivarla. A coltivare le memorie ci siamo forse ancora, tu, tua madre, e io, tu per temperamento, io e forse tua madre per temperamento e perché nella nostra vita presente non c'è nulla che valga $i$ luoghi e gli attimi incontrati lungo il percorso. Mentre io li vivevo o li guardavo, quegli attimi o quei luoghi, essi avevano uno straordinario splendore, ma perché io sapevo che mi sarei curvato a ricordarli. Mi ha sempre addolorato profondamente che Michele non volesse o non potesse conoscere questo splendore, e andasse avanti senza mai voltare la testa indietro. Credo però che lui senza saperlo contemplasse questo splendore dentro di $\mathrm{me}^{16}$.

La Ginzburg sottilmente modifica la triade femminile (Adriana, Mara e Angelica), inserendovi al posto di Mara la figura di Osvaldo, che rivela finalmente una particolare attenzione femminile legata ai sentimenti. Il modello di Antigone si sposta ora sulla figura di Osvaldo, che ripercorre i luoghi di Michele, piangendo sulla presunta e ideale tomba del ragazzo. È lui che ne recupera l'ultimo possibile ed elementare barlume di vita. Ed è lui che teorizza sul valore salvifico della memoria, secondo una logica che recupera ma anche supera quella con cui Adriana attribuisce agli oggetti un significato affettivo. Durante la vita, vi sono dei momenti che hanno uno straordinario splendore, il cui ricordo diviene salvifico. La Ginzburg sembra così recuperare la suggestione proveniente dal mondo proustiano, che lei conosce bene dal momento che è una delle prime traduttrici della Recherche. Se torna qui il tema proustiano della memoria, viene però ricondotto al suo livello più elementare, senza il fasto strutturale che ha in Proust. La speranza finale di Osvaldo risiede proprio in questo: egli confida che la sua presenza abbia permesso a Michele di recuperare - in punto di morte - la memoria della sua vita. Ma Ginzburg attribuisce al discorso finale di Osvaldo un valore di salvezza. Questo personaggio in realtà ha seguito da vicino tutte le vicende della famiglia, è un testimone silenzioso ma attivo. In lui il mondo

\author{
15. Ibid. \\ 16. Ibid., pp. 155-156.
}


maschile raggiunge un punto di vicinanza con quello femminile, anche se in realtà dobbiamo parlare più di una tensione con il femminile che non contiene niente di drammatico ma si pone come vera differenza. La tensione, che si mostra all'inizio con la madre Adriana, poi con la sorella Viola, lentamente si attenua. Ginzburg fa di Osvaldo un uomo che si pone su un piano comportamentale ed emotivo molto diverso da quello dei personaggi maschili a cui ci ha abituato la cultura italiana. La sua malinconia, l'aria rassegnata con cui sembra affrontare ogni momento, sembrano il risultato di una vitalità che è stata smorzata. Osvaldo è un padre, ma un padre che trova una collocazione all'ombra e non al centro del mondo virile. Malgrado questo, è proprio da lui che viene riscattato il vuoto di maschilità che percorre tutto il romanzo ${ }^{17}$. Siamo a grande distanza dal mondo degli omosessuali proustiani, che sono i portatori di segreti indicibili, tali da rendere spesso vani gli sforzi del narratore per decifrarli. Ma siamo anche lontani dalla sessualità maschile spesso esibita nei romanzi vicini a quello della Ginzburg, per esempio in Parise, per non parlare di Pasolini, con cui la Ginzburg instaura una sfida intellettuale continua. Anche se volessimo pensare a Petrolio, e al personaggio di Carlo che subisce un ribaltamento del genere da maschile a femminile, con la conseguente perdita di limiti al desiderio sessuale, non troveremmo niente di simile alla configurazione della sfera dell'intimità che compare con Osvaldo. La discrezione e la fermezza di Osvaldo ne fanno quasi un unicum nella nostra letteratura. In lui l'omosessualità prende la forma di una "piccola virtù".

Marco Antonio BAzzocchi Università di Bologna

17. Per un inquadramento del romanzo all'interno dell'opera saggistica della Ginzburg si può vedere RizzAreldi Maria, Gli arabeschi della memoria. Grandi virtù e piccole querelles nei saggi di Natalia Ginz̧urg, Catania, C.U.E.C.M., 2004. 